\title{
Políticas e inovação em atenção à saúde mental: limites ao descolamento do desempenho do SUS
}

\author{
Policies and Innovation in mental healthcare: \\ limits to decoupling from the performance of the SUS
}

José Mendes Ribeiro ${ }^{1}$ Aline Inglez-Dias ${ }^{1}$
${ }^{1}$ Escola Nacional de Saúde Pública Sérgio Arouca, Fundação Oswaldo Cruz. Rua Leopoldo Bulhões 1480, Manguinhos. 21041-210 Rio de Janeiro RJ.ribeiro@ensp.fiocruz.br

\begin{abstract}
We studied Brazilian policies on mental health with respect to normative, supply and demand and financing aspects. We concluded that the sustainability of innovations in psychiatric reform depends on enhanced financing and integration with primary care community services, on the overall performance of SUS and the reduction of autonomous and exclusive services in primary care. There is high and rising pressure in demand for services measured in DALY and the incidence of disease. The reduction observed in psychiatric beds was accompanied by the systemic reduction, though with selective reduction for psychiatric hospitalizations. CAPS services have institutional limits due to the model adopted of direct public administration and local government capacity. Secondary data available show that: (i) SUS has a virtual monopoly on general outpatient and hospital services; (ii) mental health specialists belong mostly to SUS; (iii) most mental health services are outpatient services; (iv) few CAPS have day-bed services available; and ( $v$ ) there is reduced federal financing for these innovations.
\end{abstract}

Key words Psychiatric reform, Mental health, Primary care
Resumo Analisamos a política brasileira de saúde mental quanto aos aspectos normativos, de demanda, de oferta e de financiamento. Concluímos que a sustentabilidade das inovações da reforma psiquiátrica depende de melhor financiamento $e$ de integração dos serviços comunitários à rede de atenção primária, do desempenho geral do SUS e da redução de serviços exclusivos e autônomos na atenção primária. Existe elevada e crescente pressão de demanda medida em DALY e carga de doença. A redução dos leitos psiquiátricos observada acompanhou a redução sistêmica, porém com redução seletiva para as internações psiquiátricas. Os CAPS apresentam limites institucionais devido ao modelo adotado de administração pública direta e da capacidade de governo municipais. Dados secundários disponíveis revelam que: (i) o SUS tem poder quase monopsônico em serviços ambulatoriais e hospitalares em geral; (ii) os profissionais de saúde mental são predominantemente vinculados ao SUS; (iii) os serviços de saúde mental têm característica predominantemente extra-hospitalar; (iv) um número residual de CAPS dispõem de leitos diários; e (v) o financiamento federal para estas inovações é reduzido.

Palavras-chave Reforma psiquiátrica, Saúde mental, Atenção primária 


\section{Introdução}

A reforma psiquiátrica brasileira expressa um sólido movimento de ideias que evoluiu como movimento político e inovador. Afora o elo com movimentos semelhantes em países como a Itália, aqui seu desenvolvimento ocorre no tempo político da redemocratização do país e da reforma sanitária que originou o Sistema Único de Saúde (SUS). O ideário da reforma sanitária e a organização do novo sistema foi amplamente discutida por lideranças setoriais ${ }^{1,2}$. As diretrizes políticas da reforma sanitária ganharam contornos normativos na Constituição de 1988, a qual representou um novo pacto social de caráter redistributivo no país. Proteção social, reforma sanitária e reforma psiquiátrica são sinérgicas e convergentes. Destacamos a mútua dependência entre o sucesso do novo modelo de atenção em Saúde Mental (SM) e o desempenho do SUS. Por outro lado, discutimos os riscos e ameaças à sustentabilidade do modelo. Dependência do financiamento dos governos municipais, baixa integração à rede de atenção primária, natureza organizacional dos CAPS na forma de administração direta e financiamento inadequado representam as principais fragilidades do novo modelo substitutivo do sistema manicomial discutidas neste artigo.

Aspectos históricos da reforma psiquiátrica e seus fundamentos são bastante discutidos na literatura setorial, assim como a crítica ao modelo de atenção manicomial ${ }^{3}$. A transformação em agenda governamental pode ser constatada em documentos do Ministério da Saúde (MS) ${ }^{4,5}$ e se traduziu em Leis e Portarias, ganhando contornos normativos. Após tramitação no Legislativo desde 1989, a aprovação da Lei 10.216 de 6 de abril de 2001 representou um marco no processo político. Conhecida como Lei Paulo Delgado ${ }^{6}$, define o direito da pessoa portadora de transtornos mentais de ser tratada em serviços comunitários de saúde mental e restringe fortemente a internação destes pacientes.

Este aparato normativo determina a disseminação de Centros de Atenção Psicossocial (CAPS) como eixo estratégico do novo modelo. Há um reconhecimento de que o sucesso da reforma dependa da adoção de novas práticas clínicas e terapêuticas ${ }^{7}$. No entanto, ao longo de seu desenvolvimento, o movimento não foi isento de conflitos com associações civis (hospitais, familiares) e de restrições aos mecanismos de desospitalização. Ao longo de sua implementação, a noção de desospitalização orientada ao ataque ao manicômio ganhou contornos adicionais na forma de redução gradual de leitos psiquiátricos. Este processo passa a combinar o uso de leitos em hospitais gerais e de mecanismos de atenção diária de diversos níveis nos CAPS.

O novo modelo busca ganhar em qualidade e acolhimento a esta clientela, em contraposição ao obsoleto e agressivo modelo centrado no manicômio. Apesar da relevância da reforma psiquiátrica, existe uma frágil cultura avaliativa no país quanto ao impacto e aos resultados desta política. Predominam estudos de caso, ensaios e análises da política fundamentadas elaboradas no âmbito do movimento de ideias. Estudo recente $^{8}$ destacou a distribuição regional desigual dos recursos e o crescimento da população como lacunas importantes, mas não oferece evidências de sucessos ou fracassos do novo modelo.

Analisamos aspectos normativos, estimativas de demanda e perfil da oferta de serviços na atenção à SM. Assinalamos que o sucesso do modelo inovador depende do desempenho dos serviços gerais do SUS e destacamos os limites de um descolamento da reforma psiquiátrica frente aos serviços públicos gerais. Isto implica que o atendimento a esta clientela deve envolver de modo intensivo a rede de atenção primária e a presença de especialistas em saúde mental. Parece inevitável, à luz do financiamento setorial e do ritmo de expansão da atenção primária, que os CAPS se configurem cada vez mais em serviços especializados de referência para os casos mais graves e críticos.

\section{Estrutura de serviços do SUS e limites ao descolamento das políticas de saúde mental}

A saúde pública no Brasil se organizou, desde a criação do MS em 1953, por meio de ações verticais e orientadas a problemas específicos. O modelo tradicional focalizou ações sobre grandes endemias rurais e se expandiu para o controle da tuberculose, do câncer e da hanseníase, além da própria saúde mental. Este padrão evoluiu para outras ações programáticas orientadas a doenças crônicas e não transmissíveis. De forma cada vez menos verticalizada, atravessou a eclosão da epidemia de AIDS e não cessou de se reproduzir por diversas ações de saúde, como na atenção às mulheres, crianças, idosos, deficientes e de doenças transmissíveis como a dengue.

No padrão focalizado que predominou até a formação do SUS a população formalmente inserida no mercado de trabalho utilizava os servi- 
ços previdenciários e a não formalizada dependia dos serviços estaduais e municipais. O governo federal elegia prioridades e, na falta de um esquema abrangente de proteção social, atuava junto às populações pobres e vulneráveis para minorar ou resolver os principais agravos. Esta configuração reflete o que Santos ${ }^{9}$ denominou como cidadania regulada, na qual o acesso aos serviços sociais é delimitado pela posição do indivíduo no mercado de trabalho. Este tipo de arranjo, embora em novo formato, foi preservado no Brasil após o SUS, mantendo sua conotação fragmentada entre sistema público e plano privado pré-pago.

Com o SUS, as principais características dos programas se alteraram. A epidemiologia social e diversas formas de conceber a ação programáti$\mathrm{ca}^{10}$ influenciaram o debate setorial. Os programas perdem a orientação focal, envolvem protocolos de atenção integral e se articulam cada vez mais à rede pública de atenção primária e hospitalar. Cada vez mais são definidos na forma de guidelines e protocolos de atenção, clínicos ou de planejamento. Logo, as inovações em políticas, para serem sustentáveis e não restritas a pilotos e ilhas de excelência, se tornam coladas e mais dependentes da dinâmica da política pública.

Por sua vez o sistema de saúde brasileiro prossegue fragmentado e o acesso aos serviços é mediado pela renda individual. A universalização excludente ${ }^{11}$ se caracterizou, com a criação do SUS, por uma troca de clientelas, com a migração das camadas de maior renda e de maior articulação corporativa para os planos privados pré-pagos. Este processo se intensificou com o racionamento da oferta de serviços públicos hospitalares em resposta aos problemas de financiamento do SUS e consolidou este tipo de segmentação ${ }^{12}$.

Em um sistema público subfinanciado, a transferência de recursos de atenção hospitalar para os ambulatórios em nome da eficiência sistêmica pode levar a um grave quadro de escassez e barreiras de acesso aos serviços mais complexos. As inovações em saúde mental orientadas à atenção comunitária dependem destes movimentos serem altamente coordenados e capazes de compensar lacunas de provisão de serviços, como ocorre no SUS. A desospitalização preconizada pode ser prejudicada caso se insista em organizar uma rede de SM em bases exageradamente autônomas.

Além disso, a transição dos programas focais para sistemas integrados e protetores não se completou no Brasil. A estrutura burocrática do MS se manteve por meio de programas verticalmente estruturados e superpostos aos das se- cretarias estaduais e municipais. A competição burocrática afeta determinados programas em detrimento de outros. A burocracia pública opera como arena altamente competitiva e sensível aos grupos de maior ativismo e com sólidas conexões societárias. Os atores da reforma psiquiátrica operam neste ambiente institucional.

\section{Modelo de atenção à saúde mental}

$\mathrm{O} \mathrm{MS}^{5}$ tem discutido as perspectivas da atenção comunitária e hospitalar em saúde mental sob a ótica da reforma e reflete a experiência de países dotados de sistemas sanitários sólidos. Um sistema de atenção comunitária, ambulatorial e hospitalar foi sistematizado no World Health Report $^{13}$ e a reforma psiquiátrica brasileira está alinhada a estas soluções.

A lacuna quanto a profissionais habilitados e estimativas de gastos para superar o chamado mental health gap têm sido amplamente discutidas pela Organização Mundial de Saúde (OMS). Estudo recente ${ }^{14}$ estimou que 93\% dos países de renda baixa e 59\% daqueles de renda média apresentavam escassez de psiquiatras para suprir as necessidades do novo modelo.

O modelo reformador preconiza a substituição progressiva dos hospitais por serviços cuja complexidade, em termos de equipamentos, é menor em troca de maior complexidade na abordagem aos pacientes e no suporte às suas famílias. Propõe-se a troca do ambiente hospitalar, com maior escala de atendimento e acesso a recursos de emergência, por um ambiente comunitário e ambulatorial mais adaptado às necessidades individuais e familiares - troca-se escala por qualidade. Há maior complexidade e intensidade no trabalho profissional em saúde mental. A substituição regulada da atenção hospitalar pela atenção centrada em ambulatórios e comunidades envolve a criação de novos serviços (CAPS, residência terapêutica, e outros dispositivos de atenção psicossocial).

\section{Aspectos da demanda}

sobre a atenção em saúde mental

Revisão da literatura demonstra que os transtornos mentais representam um problema de saúde global. No entanto, quanto à demanda por serviços, foram constatados alguns problemas. Há uma elevada heterogeneidade de informações sobre a epidemiologia dos transtornos mentais, que variam desde os comuns ${ }^{15}$ - transtornos 
depressivos, de ansiedade e somatoformes - aos mais graves e incapacitantes ${ }^{16}$ - esquizofrenia e psicoses não afetivas, transtorno bipolar, depressão maior e transtorno do pânico. Um número menor de estudos incluem transtornos relacionados à dependência de substancias psicoativas.

Outro cuidado a ser tomado, especialmente quando se trata de inquéritos populacionais, diz respeito ao estigma. Transtornos mentais são estigmatizados em muitas culturas, apesar das inúmeras iniciativas e esforços de instituições internacionais e governamentais para mudar tal cenário $^{17}$. Assim, é possível que sejam sub-reportados por participantes desses inquéritos.

Quanto aos estudos realizados em serviços específicos de saúde mental, a demanda calculada não traduz, necessariamente, a demanda que acessou estas unidades pelo fato de muitos profissionais não estarem aptos a reconhecer sintomas específicos e efetuarem o encaminhamento adequado. Estima-se que apenas um caso de transtorno mental em vinte observados na clínica geral seja encaminhado para médico ou serviços especializados ${ }^{15}$. Isto pode ser corroborado pelo elevado consumo de psicotrópicos prescritos pela população em geral ${ }^{15,18}$. Transtornos como a depressão, onde com frequência o paciente procura o serviço de saúde reportando sintomas físicos inespecíficos, acabam subdiagnosticados e, consequentemente, subtratados. Estima-se que menos de um quarto desses casos sejam diagnosticados e tratados corretamente ${ }^{19}$, comprometendo uma noção mais acurada sobre as reais necessidades desta clientela. Isso reforça a ideia de que profissionais especializados devem estar mais presentes nos serviços gerais de atenção em saúde.

Globalmente, transtornos mentais e neurológicos foram responsáveis por $12 \%$ do total de Anos de Vida Ajustados por Incapacidade (DALY, em inglês) no ano $2000^{13}$. Entre os principais diagnósticos foram listados os transtornos depressivos, a esquizofrenia, os resultantes do abuso de substância, a epilepsia, o retardo mental, os transtornos da infância e adolescência e a doença de Alzheimer. Estima-se que, até 2020, a carga dessas doenças terá atingido o patamar de $15 \%{ }^{13}$.

Estudo sobre carga de doença relacionada à saúde mental ${ }^{16} \mathrm{em}$ países em desenvolvimento considerou quatro tipos principais de transtornos: esquizofrenia e psicoses não afetivas, transtornos afetivos bipolares, transtorno depressivo maior e transtorno de pânico. A escolha por tais transtornos se deveu não apenas à carga de doença atribuída aos mesmos, mas também à disponibilidade de dados para avaliação de custo-efeti- vidade de tratamentos associados. Inquéritos epidemiológicos em diversos países apresentaram prevalência ao longo da vida variando de 0,3 a $1,6 \%$ para esquizofrenia, 0,1 a $2,0 \%$ para transtornos de humor, 4,2 a $17,0 \%$ para transtornos depressivos (depressão maior e distimia) e 2,2\% a $28,5 \%$ para transtornos de ansiedade ${ }^{16}$. No Brasil, a prevalência de transtornos mentais graves (psicoses, neuroses graves, transtornos de humor graves e deficiência mental com grave dificuldade de adaptação) na população é estimada em cerca de 3\%. Assim, os números apontam para uma demanda de cerca de cinco milhões de pessoas, que necessitariam de cuidados contínuos e intensivos no âmbito da saúde mental. Além disso, em torno de 10 a $12 \%$ da população sofre de transtornos menos graves, mas que também necessitam de algum cuidado em saúde mental, na forma de consulta médico-psicológica, aconselhamento, grupos de orientação e outras formas de abordagem ${ }^{4}$.

Segundo dados de um inquérito ${ }^{20}$ realizado no Brasil, entre adultos a prevalência de transtornos mentais ao longo da vida, no último ano e no último mês foi, respectivamente de $45,9 \%$ / $26,8 \% / 22,2 \%$. Os transtornos mais prevalentes ao longo da vida foram: dependência à nicotina $(25,0 \%)$; transtornos do humor (18,5\%); episódios depressivos como o tipo mais comum de transtorno de humor $(16,8 \%)$; transtornos de ansiedade $(12,5 \%)$; transtornos somáticos (6,0\%); e dependência/abuso de álcool (5,5\%). Outro estudo ${ }^{21}$ aponta para taxas de prevalência de transtornos mentais (clinicamente significativos) no país variando de $10,2 \%$ a $18,0 \%$. Os transtornos neuropsiquiátricos foram responsáveis em 1998 no Brasil 19,0\% dos DALY no ano de 1998. A maior parte da carga originada de transtornos neuropsiquiátricos se deveu à depressão, às psicoses e aos transtornos relacionados ao consumo de álcool ${ }^{22}$.

Quanto ao uso de álcool e de outras drogas, um tema crescente na agenda política nacional, os números globais são elevados, assim como a variedade de drogas disponíveis ao consumo. Em todo o mundo, estima-se que entre 155 e 250 milhões de pessoas ( 3,5 a $5,7 \%$ da população com idade entre 15-64 anos) usaram drogas ilícitas ao menos uma vez durante o ano de $2008^{23}$. Em toda América Latina e Caribe, estima-se que ao menos 4.4 milhões de homens e 1.3 milhões de mulheres tenham sido afetados por transtornos relacionados ao uso de drogas, em algum momento de suas vidas ${ }^{24}$. Na América do Sul, embora a droga ilícita mais consumida seja a maconha, o World Drug Report de $2010^{23}$ aponta para 
um crescimento do uso de cocaína e seus derivados (especialmente o crack). Para a América do Sul, Central e Caribe estima-se que cerca de 2.7 milhões de pessoas tenham feito uso de cocaína no ano de 2008 e o Brasil pode ser apontado como seu principal mercado consumidor (900 mil usuários) na América do Sul. O Brasil apresenta a prevalência mais alta para medicamentos derivados do ópio (0,5\% da população entre 15 64 anos) e um mercado de cerca de 640 mil usuários. Assim como no restante do mundo, no Brasil é o álcool a substância que apresenta a maior carga global de agravos indesejáveis e extremamente dispendiosos, acometendo indivíduos em todas as áreas de suas vidas.

No entanto, os números relativos ao uso de drogas (especialmente as ilícitas) em toda América Latina e Caribe devem ser interpretados com cuidado, já que a quantidade e a qualidade de informações epidemiológicas são consideradas um significativo desafio ${ }^{24}$. No Brasil os levantamentos nacionais são escassos ${ }^{25,26}$ e a literatura disponível é composta por estudos pontuais, predominantemente nas regiões sul e sudeste, que não refletem a realidade do uso de drogas no país como um todo.

Esta demanda difusa vem sendo historicamente preenchida por instituições outras que não as preconizadas por políticas públicas. Até o ano de 2002, não havia sequer uma política de saúde nacional e as iniciativas governamentais estavam restritas a poucos serviços hospitalares ou ambulatoriais, geralmente vinculados a universidades ${ }^{4}$.
Apesar da grande variabilidade nas estimativas de impacto dos transtornos mentais, uma pressão de demanda sobre os serviços de saúde da ordem de 12\% (como apontado em DALY) está claramente desbalanceada frente aos recursos atualmente disponíveis no país. Além disso, como nos estudos mais localizados aqui citados, a demanda estimada pode ultrapassar as estimativas em DALY. Tamanha carga de doença exige um volume de recursos financeiros, de pessoal especializado, de instalações, de equipamentos e de medicamentos em escalas distantes da oferta vigente do SUS. É esperado que a abertura de novos serviços seja acompanhada de crescente sobrecarga de demanda.

\section{Oferta de serviços do SUS e em saúde mental}

Uma análise sobre a evolução da oferta de serviços de atenção em SM envolve o conhecimento dos protocolos para a organização de um sistema preconizado, do financiamento da política e dos serviços públicos disponíveis. As fontes aqui utilizadas são provenientes do MS ou de pesquisas realizadas pelo IBGE. Embora numerosos e diversificados, os dados obtidos não permitem uma avaliação completa de resultados. No entanto, é possível delinear o descompasso entre a demanda e a oferta de serviços de atenção primária e hospitalar tanto no SUS em geral, como em SM.

Os dados da Tabela 1 mostram elementos importantes na assistência hospitalar do setor

Tabela 1. Internações hospitalares (frequências absolutas e relativas), SUS, grandes grupos de CID, 19952010 .

\begin{tabular}{rrrrrrrrr}
\hline Ano & $\begin{array}{c}\text { Grav parto } \\
\text { puerp }\end{array}$ & $\begin{array}{c}\text { D ap } \\
\text { circulatório }\end{array}$ & $\begin{array}{c}\text { D infece } \\
\text { parasitárias }\end{array}$ & $\begin{array}{c}\text { Causas } \\
\text { externas }\end{array}$ & $\begin{array}{c}\text { Neoplasias } \\
\text { Tr mentais } \\
\text { compor }\end{array}$ & $\begin{array}{c}\text { Demais } \\
\text { causas }\end{array}$ & Total \\
\hline 1995 & 3.269 .395 & 1.260 .274 & 1.112 .123 & 735.769 & 400.764 & 438.334 & 5.429 .541 & 12.646 .200 \\
& 25,85 & 9,97 & 8,79 & 5,82 & 3,17 & 3,47 & 42,93 & $100,00 \%$ \\
1998 & 2.925 .596 & 1.014 .922 & 874.949 & 608.269 & 342.550 & 426.602 & 5.521 .868 & 11.714 .756 \\
& 24,97 & 8,66 & 7,47 & 5,19 & 2,92 & 3,64 & 47,54 & $100,00 \%$ \\
2001 & 2.810 .974 & 1.163 .808 & 945.762 & 696.886 & 390.859 & 388.722 & 5.359 .343 & 11.756 .354 \\
& 23,91 & 9,90 & 8,04 & 5,93 & 3,32 & 3,31 & 45,59 & $100,00 \%$ \\
2004 & 2.645 .411 & 1.205 .067 & 962.876 & 755.826 & 585.551 & 294.730 & 5.043 .422 & 11.492 .883 \\
& 23,02 & 10,49 & 8,38 & 6,58 & 5,09 & 2,56 & 43,89 & $100,00 \%$ \\
2007 & 2.486 .305 & 1.157 .509 & 915.763 & 832.858 & 640.325 & 290.079 & 5.007 .257 & 11.330 .096 \\
& 21,94 & 10,22 & 8,08 & 7,35 & 5,65 & 2,56 & 44,19 & $100,00 \%$ \\
2010 & 2.377 .590 & 1.153 .191 & 1.021 .300 & 929.240 & 604.809 & 285.736 & 4.985 .898 & 11.357 .764 \\
& 20,93 & 10,15 & 8,99 & 8,18 & 5,33 & 2,52 & 43,90 & 100,00 \\
\hline
\end{tabular}

Fonte: Ministério da Saúde/DATASUS - Sistema de Informações Hospitalares do SUS. Obs: agregamos diversos grupos de CID ao de "demais causas" (XXI) original na composição desta tabela. 
público. Quanto às internações, há diminuição progressiva e sustentada no total realizado. Estudo ${ }^{12}$ demonstra que esta redução foi seletiva para prestadores privados com fins lucrativos. Este movimento foi apenas parcialmente compensado pela expansão dos serviços hospitalares mantidos pelos governos municipais. Dados recentes do IBGE $^{27}$ confirmam este movimento.

A redução dos leitos psiquiátricos no período é convergente a esta evolução. O número de internações por transtornos psiquiátricos e comportamentais foi reduzido significativamente (de $438.334 \mathrm{em} 1995$ para $285.736 \mathrm{em} 2010$ ). No entanto, há uma seletividade neste processo, pois a participação percentual destas internações diminuiu de modo consistente de 3,47\% (1995) a 2,52\% (2010). Movimento contrário, com aumento absoluto e proporcional de internações, foi observado para "causas externas" e "neoplasias" e refletem a urbanização e a transição epidemiológica. É importante lembrar os efeitos de aumento populacional e de crescimento da população idosa quando se analisa estas séries históricas e sua apreciação reforça os aspectos restritivos da oferta de serviços.

Tomando o conjunto da atenção hospitalar no país, dados da AMS $2009^{27}$ mostram que número total de leitos variou pouco - de 443.888 (1979) a 431.996 (2009) - havendo aumento sustentado dos leitos de propriedade estatal e mudanças no setor privado. Como o total de internações para todos os grupos diminui conforme a redução de leitos do SUS, sugerimos que não houve ganhos significativos em eficiência por melhoria de gestão e incorporação tecnológica. O total de estabelecimentos de saúde de todos os tipos foi de 94.070 unidades, das quais 49.753 de propriedade estatal federal, estadual e municipal $(55,3 \%)$ e 42.049 unidades de propriedade privada (44,7\%). Dentre os estabelecimentos privados, um total de 11.380 prestavam serviços ao SUS. Temos assim 61.133 estabelecimentos prestadores do SUS correspondendo a $65,0 \%$ da oferta. A participação dos estabelecimentos estatais é proporcionalmente maior no atendimento ambulatorial $(69,8 \%)$, a ser acrescida de $39,4 \%$ de prestadores privados do SUS. Os meios diagnósticos e terapêuticos estão concentrados fortemente no setor privado autônomo $(59,0 \%)$ e privado prestador do SUS $(31,9 \%)$. A área hospitalar é dominada pelos estabelecimentos de propriedade estatal $(41,3 \%)$ e privados contratados pelo SUS (39,4\%). Logo, o SUS é o protagonista nos serviços ambulatoriais e hospitalares com poder quase monopsônico.

No caso da atenção em SM as inovações exigem equipes multidisciplinares e profissionais especializados (Tabela 2). Os parâmetros usuais da Portaria 1.101/2002 28 se remetem às proporções de consultas e procedimentos realizados e não permitem responder a questão da adequação da distribuição de profissionais observada. O número de psiquiatras (12.207) nos sistemas público e privado, embora elevado, pode representar uma restrição importante devido à distribuição nacional desigual, a uma dedicação em tempo integral restrita (1.955) e à disponibilidade para fixação em serviços de dedicação rotineira e quase exclusiva. Esta característica do trabalho médico é predominante no país.

A organização dos CAPS foi atualizada pela Portaria $336 / 2002^{29}$ do MS a partir da regulamentação original, efetuada em 1992, e as equipes pro-

Tabela 2. Pessoal de nível superior em estabelecimentos de saúde, ocupação e jornada de trabalho, Brasil, 2009.

\begin{tabular}{lcrrr}
\hline \multicolumn{1}{c}{ Profissão } & Integral & Parcial & Indefinida & Total \\
\hline Médicos (exceto Psiquiatras) & 141.572 & 343.621 & 140.942 & 626.135 \\
Enfermeiro & 82.286 & 76.066 & 4.747 & 163.099 \\
Odontólogo & 29.840 & 56.166 & 8.130 & 94.136 \\
Outros & 19.406 & 28.463 & 4.466 & 52.335 \\
Fisioterapeuta & 13.148 & 30.855 & 6.265 & 50.268 \\
Bioquímico/farmacêutico & 18.664 & 18.596 & 2.728 & 39.988 \\
Psicólogo & 7.118 & 18.512 & 2.739 & 28.369 \\
Nutricionista & 6.613 & 10.970 & 1.634 & 19.217 \\
Assistente social & 7.785 & 10.203 & 598 & 18.586 \\
Psiquiatra & 1.955 & 8.391 & 1.861 & 12.207 \\
Total & 328.387 & 601.843 & 174.110 & 1.104 .340 \\
\hline
\end{tabular}

Fonte: IBGE, Pesquisa de Assistência Médico Sanitária 2009 
fissionais mínimas são definidas segundo o porte dos municípios. De acordo com os estratos populacionais definidos chegamos à distribuição segundo o tipo de CAPS e o porte municipal. Os CAPS I (1.230 municípios com $20 \mathrm{mil} \mathrm{a} 70 \mathrm{mil}$ habitantes) devem contar com um médico psiquiatra ou profissional treinado em saúde mental. Os CAPS II (280 municípios com 70 mil a 200 mil habitantes) devem ter ao menos um médico psiquiatra que responda pelo serviço. Os CAPS III (134 municípios com mais de 200 mil habitantes) devem funcionar em regime integral e contar com leitos de repouso, e ter dois psiquiatras. Uma contagem simplificada aponta a necessidade de ao menos 1.778 psiquiatras em regime de dedicação integral. Segundo esta base simplificada de incorporação profissional, haveria disponibilidade no país para a expansão do modelo.

Esta configuração envolve uma frágil incorporação de profissional especializado. A exigência de psiquiatras nestas equipes é mínima. A presença do psicólogo, um tipo de especialista já qualificado à atenção em SM, é facultativa e diluída dentre um grupo mais geral de profissionais de nível superior. A própria Portaria 154/2008 ${ }^{30}$ do MS estabelece para os Núcleos de Atenção em Saúde da Família uma configuração mínima para SM semelhante a dos CAPS. A baixa incorporação de especialistas para lidar com pacientes graves e com a demanda rotineira é uma ameaça à sustentabilidade do modelo que precisa ser melhor estudada. Esta restrição também afeta o uso intensivo da rede de atenção primária em SM.

Meios mais adequados de estimar parâmetros para profissionais de SM têm sido discutidos. Estudo recente ${ }^{14}$ estimou para a Argentina a necessidade, por exemplo, de 758 psiquiatras em tempo integral. Uma projeção para a base populacional brasileira implicaria numa demanda de cerca de 3.747 psiquiatras em tempo integral.

Dados do CNES mostram que 77,9\% dos psiquiatras e 69,3\% dos psicólogos estão vinculados ao SUS (Tabela 3 ). As proporções são, respectivamente, 0,1 e 0,2 por mil habitantes. A PNAD/2008 do IBGE $^{31}$ mostrou que dentre os 13.513.509 entrevistados que referiram internação no último ano, um total de 4.018 .489 (29,7\%) possuía algum tipo de cobertura de plano de saúde. No caso das internações psiquiátricas, esta proporção foi de $21,7 \%$. Isto sugere que esta clientela é altamente dependente do SUS e os vínculos profissionais seguem este perfil.

Dados do IBGE ${ }^{32}$, de 2005, identificaram 3.149 estabelecimentos com atendimento em saúde mental, dos quais $70,5 \%$ são da administração direta. Esta forma organizacional é menos adaptativa e ágil na gestão de pessoal, aquisição de equipamentos e realização de obras ${ }^{33}$. O Programa de Saúde da Família, por exemplo, apresenta alta participação de organizações sociais, fundações e associações de direito privado, entre outros entes, sob contrato de metas e resultados. Além disso, há forte dependência da capacidade de governo do nível municipal onde estão localizados 64,1\% dos serviços de SM. A dependência de serviços municipais em locais de baixa capacidade institucional, associada ao modelo da administração direta, podem representar sérios obstáculos às inovações preconizadas.

Os serviços de saúde mental (Tabela 4) são principalmente extra-hospitalares, vinculados ao SUS e fortemente ambulatoriais. Isto está de acordo com o modelo preconizado. O fato de os dados sobre hospital-dia mostrarem uma cobertura de apenas $11,8 \%$ não deve ser visto como uma

Tabela 3. Resumo de número de vínculos profissionais segundo categorias selecionadas, Brasil, dezembro de 2009.

\begin{tabular}{lrrr}
\hline \multicolumn{1}{c}{ Categoria } & \multicolumn{1}{c}{ Total } & \multicolumn{1}{c}{ SUS } & Outros $^{* *}$ \\
\hline Médicos - Clínico Geral & 176.407 & 149.021 & 27.386 \\
Médicos de Família & 35.419 & 35.316 & 103 \\
Médicos - Psiquiatra & 16.125 & 12.566 & 3.559 \\
Médicos - Outros & 615.149 & 403.496 & 211.653 \\
Enfermeiro & 144.260 & 132.313 & 11.947 \\
Fisioterapeuta & 52.577 & 33.321 & 19.256 \\
Farmacêutico & 43.202 & 35.511 & 7.691 \\
Psicólogo & 38.533 & 26.721 & 11.812 \\
Assistente social & 22.790 & 21.950 & 840 \\
\hline
\end{tabular}

Fonte: CNES. * contagem efetuada por vínculos de emprego registrados e admite-se multiplicidade. ${ }^{*}$ inclui vínculos de profissionais não disponíveis ao SUS

Tabela 4. Número de estabelecimentos com atendimento em saúde mental segundo tipo de serviços oferecidos $(n=3.146)$

\begin{tabular}{lcc}
\hline & Não & Sim \\
\hline Hospital dia & 88,2 & 11,8 \\
Internação & 71,5 & 28,5 \\
Urgência/emergência & 68,8 & 31,2 \\
Atendimento SUS & 12,6 & 87,4 \\
Atendimento ambulatorial & 13,5 & 86,5 \\
Visita domiciliar & 47,0 & 53,0 \\
\hline
\end{tabular}

Fonte: IBGE - Pesquisa de Assistência Médica Sanitária (AMS) 2002 
lacuna na oferta. Os normativos recentes no contexto da reforma psiquiátrica deixaram de preconizar este tipo de dispositivo como solução na atenção aos transtornos mentais. Portanto, o foco deve ser concentrado na capacidade dos CAPS de atender aos pacientes graves ou em situação aguda por meio de dispositivos de atenção diária.

A análise dos gastos no setor público de saúde no Brasil traz algumas dificuldades importantes. As principais são o cofinanciamento entre os três níveis de governo (com estruturas contábeis diversas), o financiamento não alinhado aos custos reais e as fragilidades dos sistemas de informações públicos. No caso de programas que apresentam sinergias com a oferta geral do sistema, a falta de registro individualizado da passagem dos usuários segundo diagnósticos e procedimentos realizados é um empecilho importante. Para as internações (onde a informação é mais completa), a avaliação de resultados é viável. Para o atendimento ambulatorial, que representa a base da reforma, as informações em escala nacional são insuficientes.

Para contornar em parte estes problemas, o financiamento da SM foi analisado segundo as transferências do MS a título de incentivos para Estados e Municípios. Estes incentivos refletem prioridades políticas do MS. Os demais incentivos estão distribuídos nas transferências não vinculadas à tabela de pagamentos, como no Piso da Atenção Básica (PAB). Comparamos as transferências explícitas para SM com políticas estratégicas do SUS (PAB, PSF e Controle de HIV/ AIDS). Estes dados (Tabela 4) foram agrupados a partir do extrato original de 113 itens com diferentes graus de abrangência e valores, excluída a parte de medicamentos. A comparação com a política de DST/AIDS se justifica pelo caráter de descolamento inicial assinalado frente aos serviços do SUS. O agrupamento de incentivos para o PSF decorre da relevância da agenda governamental e o PAB é a principal transferência para o conjunto das atividades definidas como básicas.

Os resultados mostram a pulverização dos incentivos. Apenas dois itens se destacaram do total transferido ( $\mathrm{R} \$ 21.899 .518 .677,64)$, com valores anuais acima de cinco bilhões e representam incentivos gerais. Para o total das transferências (113 itens), a mediana foi só de R \$ 5.901.252,99 para uma média de R\$192.101.041,03. Isto demonstra a forte presença de incentivos de valores nacionais baixos.

Considerando os incentivos para os grupos selecionados (exceto o PAB) observa-se que a maior parte $(62,0 \%)$ foi para os municípios como esperado pela orientação localista do SUS. Quando observamos o perfil dos incentivos para as ações fora do grupo aqui destacado (e que refletem procedimentos de maior complexidade), a participação municipal como receptor cai para 51,0\%. Quanto maior a complexidade dos programas, menor o protagonismo municipal. O PAB fixo é transferido na quase totalidade aos municípios $(98,8 \%)$, assim como os recursos do PAB variável $(98,5 \%)$. Os recursos de HIV/AIDS $(65,2 \%)$ e SM $(61,1 \%)$ também demonstram o foco no município, porém com presença estadual relevante. Quanto à SM, as cinco modalidades de CAPS têm 100,0\% de incentivos para os municípios. Assim, a desospitalização foi confiada à desigual capacidade de governo dos municípios brasileiros. Chama a atenção os baixos valores envolvidos nas transferências aos CAPS (R\$ 96 mil a R\$2.738.000). O maior incentivo do grupo foi para o acompanhamento de pacientes de saúde mental no valor de $\mathrm{R} \$ 124.191 .723,50$ e com participação municipal de $60,8 \%$.

Procuramos observar o financiamento à SM segundo os procedimentos realizados, apesar das restrições aqui apontadas. Dados dos Cadernos de Informações em Saúde do MS para 2009 mostram que os totais pagos foram de $\mathrm{R} \$$ 13.872.562.563,74 para procedimentos ambulatoriais e R\$10.111.660.174,03 para hospitalares. Apenas dois itens ambulatoriais registram pagamentos de SM: (i) atendimento ou acompanhamento em reabilitações diversas, inclusive mental (R\$ 779.972,26); e (ii) atendimento ou acompanhamento psicossocial ( $\mathrm{R} \$ 68.094,65)$.

Estes valores, absolutamente residuais, se assemelham ao quadro observado anteriormente para os incentivos. Parece evidente que o financiamento federal em separado para a agenda de reforma psiquiátrica é reduzido e que os recursos devem ser disputados pelos empreendedores da reforma no bloco geral de pagamentos gerais do SUS.

Uma alternativa de financiamento específico ficaria por conta dos recursos estaduais e municipais que geralmente reproduzem o arranjo do MS. Logo, exceto em locais especialmente aderidos à reforma, a viabilidade do novo modelo depende ou de um elevado aumento no financiamento destas políticas ou de sua conversão radical para a estrutura formal da atenção primária. Os CAPS deveriam ter suas atividades concentradas nos pacientes mais graves e observação diária. A rede primária deve gradativamente aumentar a inclusão de profissionais de saúde mental através dos NASF, como discutido no Relatório da IV Conferência de Saúde Mental ${ }^{34}$. O documento sobre 
os 15 anos da assinatura pelo Brasil da Declaração de Caracas ${ }^{4}$ efetua um balanço político da reforma e defende que os CAPS devem ser substitutivos e não complementares aos hospitais. Dados do CNES registram 1.826 CAPS e apenas 34 deles informam disponibilidade de leitos para internação, o que pode traduzir desde problemas no adequado registro do leito ou numa forte restrição na aplicação do modelo substitutivo. Documento do $\mathrm{MS}^{5}$ assinala a existência de 0,66 CAPS/ 100 mil habitantes para uma meta de 1/100 mil. A lacuna na provisão de serviços necessários ao desenvolvimento é reconhecida pelo próprio MS como um "desfinanciamento da saúde" que poria em risco a sua sustentabilidade, especialmente para a expansão dos CAPS III.

\section{Considerações finais}

Após dez anos de implantação da Lei Paulo Delgado, um balanço de riscos à sustentabilidade das inovações introduzidas foi delineado em nosso artigo. A agenda política ganhou novos contornos ao longo deste período. A desospitalização nos primórdios da reforma representou uma reação às condições desumanas observadas em muitos manicômios. Em seguida, a desospitalização se tornou um processo regulado e substitutivo. A atenção centrada nos hospitais foi substituída por uma ênfase em equipamentos comunitários representados pelos CAPS. Os CAPS devem atuar como serviços especializados para o atendimento a pacientes com diferentes níveis de gravidade. Um sistema abrangente de porta de entrada deve incluir a rede de atenção primária disponível e profissionais de saúde mental devem participar ativamente destes serviços. Com relação à atenção aos pacientes graves e em crise, a resposta adequada está no fortalecimento dos CAPS II e III e na disponibilidade de leitos de observação diária. A parte mais crítica do sistema de atenção em saúde mental parece, no entanto, estar no acesso aos primeiros serviços e com qualidade. Tendo em vista as decisões políticas adotadas no país, a expansão de cobertura destes serviços terá de ocorrer, a nosso ver, no escopo do que se denomina no SUS como "atenção básica”. Recomendações da IV Conferência em Saúde Mental reforçam a necessidade do aumento da cobertura de ESF, bem como de sua articulação na atenção à SM.

Outro novo contorno da política foi a crescente participação da atenção aos usuários e dependentes de álcool e drogas. Isto ocorreu por meio de novos serviços como os CAPSad e consultórios de rua. O foco em clientelas específicas como crianças e adolescentes, população de rua e idosos se incluem como exemplos de expansão da agenda reformadora.

Os sucessos objetivos da reforma no combate às condições observadas nos manicômios e a expansão acelerada de CAPS são amplamente difundidos na imprensa e na literatura especializada. A maior articulação da atenção à SM com a atenção primária do SUS ampliaria as possibilidades de universalização destes serviços. Por outro lado, obstáculos importantes persistem como desafios políticos que esbarram nas limitações do próprio SUS e envolvem financiamento, capacidade de governo e disponibilidade de pessoal especializado.

O modelo proposto pela reforma psiquiátrica herda alguns elementos dos programas verticais caracterizados por um descolamento da política geral e combina esta tradição com os aspectos contemporâneos de descentralização dos serviços para o nível municipal e que estavam ausentes na tradição campanhista e verticalizada da saúde pública. Argumentamos que o descolamento da atenção à SM na forma de um sistema a parte na atenção primária é questionável. A magnitude da demanda, os meios diagnósticos e terapêuticos e o uso intensivo de pessoal especializado impedem uma expansão da política sem que suas ações estejam inseridas nos serviços de saúde em geral. Desta forma os CAPS deveriam atuar como serviços especializados de referência e a presença de pessoal especializado em SM deveria ser fortalecida nos serviços hospitalares, ambulatoriais e comunitários do SUS. A presença de pessoal especializado na atenção primária representa um importante item de acesso dessa clientela aos serviços. Mecanismos de controle sobre efeitos iatrogênicos em SM devem ser fortalecidos no sentido de evitar uma medicalização abusiva de casos sem indicação objetiva. Este ganho de escala deve ser procurado não apenas pela magnitude e diversidade da demanda, mas também devido aos obstáculos aqui discutidos.

A questão da inserção da atenção em saúde mental junto aos serviços de atenção primária tem sido discutida por especialistas da área para além dos problemas relacionados à iatrogenia. Destacados atores no contexto da reforma psiquiátrica, como Alves ${ }^{35}$, por motivos e caminhos distintos dos aqui considerados, preconizam, em nome do caráter comunitário do novo modelo e da integralidade prevista na reforma sanitária, a incorporação dos novos serviços de saúde men- 
tal ao que hoje é conhecido como Estratégia da Saúde da Família.

A sustentabilidade das inovações é ameaçada por problemas gerais associados ao financiamento da política de saúde. Além disso, a dependência dos governos municipais de capacidade altamente desigual na organização do modelo comunitário é uma restrição importante a nosso ver. A baixa integração à rede de atenção primária e a natureza organizacional dos CAPS, na forma de administração direta, são outros problemas discutidos que podem afetar a disseminação e o sucesso do modelo substitutivo.

\section{Colaboradores}

JM Ribeiro e A Inglez-Dias participaram igualmente de todas as etapas da elaboração do artigo.

\section{Referências}

1. Cordeiro H. Sistema Único de Saúde. Rio de Janeiro: Ayuri Editorial; 1991.

2. Cordeiro H. Descentralização, universalidade e equidade nas reformas de saúde. Cien Saude Colet 2001; 6(2):319-328.

3. Amarante P. Novos sujeitos, novos direitos: o debate em torno da reforma psiquiátrica. Cad Saude Publica 1995; 11(3):491-494.

4. Brasil. Ministério da Saúde (MS). Coordenação Geral de Saúde Mental. Reforma psiquiátrica e política de saúde mental no Brasil. In: Conferência Regional de Reforma dos Serviços de Saúde Mental: 15 anos depois de Caracas; 2005; Brasília, OPAS.

5. Brasil. Ministério da Saúde (MS). Coordenação Geral de Saúde Mental, Álcool e Drogas. Saúde Mental no SUS: as novas fronteiras da reforma psiquiátrica. Relatório de Gestão para o período 20072010. Brasília: Ministério da Saúde (MS); 2011.

6. Brasil. Ministério da Saúde. Lei 10.216 de 6 de abril de 2001. Dispõe sobre a proteção e os direitos das pessoas portadoras de transtornos mentais e redireciona o modelo assistencial em saúde mental. Diário Oficial da União 2001; 6 abr.

7. Berlinck MT, Magtaz AC, Teixeira M. A reforma psiquiátrica brasileira: perspectivas e problemas. Rev. latinoam. psicopatol. fundam. 2008; 11(1):21-27.

8. Mateus MD, Mari JJ, Delgado PGG, Almeida-Filho N, Barrett T, Gerolin J, Goihman S, Razzouk D, Rodriguez J, Weber R, Andreoli SB, Saxena S. The mental health system in Brasil: policies and future challenges. International Journal of Mental Health Systems [periódico na Internet]. 2008 [acessado 2011 out 12];2(12). Disponível em: http://www.ijmhs. com/content/2/1/12

9. Santos WG. Cidadania e Justiça: a política social na ordem brasileira. Rio de Janeiro: Campus; 1979. 
10. Schraiber LB, organizador. Programação em saúde hoje. São Paulo: Hucitec; 1990.

11. Favaret Filho P, Oliveira PJ. A Universalização excludente: reflexões sobre as tendências do sistema de saúde. Planejamento e Políticas Públicas 1990; 3:139-162.

12. Ribeiro JM. UHS development and hospital services rationing. Cien Saude Colet 2009; 14(3):771-782.

13. World Health Organization (WHO). Mental Health: new understanding, new hope. Geneva: World Health Report; 2001.

14. Scheffler RM, Bruckner TA, Fulton BD, Yoon J, Shen G, Chisholm Morris J, Dal Poz MR, Saxena S. Human resources for mental health: workforce shortage in low - and middle-income countries. Human Resources for Health Observer 2011; (8).

15. Mari JJ, Jorge MR. Transtornos psiquiátricos na clínica geral. Psychiatry On-line Brazil [periódico na Internet]. 1997 [acessado 2011 jul 27]; 2. Disponível em: http://www.polbr.med.br/ano97/tpqcm.php

16. Hyman S, Chrisholm D, Kessler R, Patel V, Whiteford H. Mental Disorders. In: Disease control priorities in developing countries. Oxford: World Bank/ Oxford University Press; 2006.

17. Weiss MG, Jadhav S, Raguram R, Vounatsou P, Littlewood R. Psychiatric stigma across cultures: local validation in Bangalore and London. Anthropology and Medicine 2001; 8(1):71-87.

18. Andrade MF, Andrade RCG, Santos V. Prescrição de psicotrópicos: avaliação das informações contidas em receitas e notificações. Rev. Bras. Cienc. Farm. 2004; 40(4):471-479.

19. Linden M, Lecrubier Y, Bellantuono C, Benkert O, Kisely S, Simon G. The prescribing of psychotropic drugs by primary care physicians: an international collaborative study. J Clin Psychopharmacol 1999; 19(2):132-140.

20. World Health Organization (WHO). Mental Health Atlas 2005. Mental Health: Evidence and Research, Department of Mental Health and Substance Abuse. Geneva: World Health Organization (WHO); 2005.

21. Mello MF, Kohn R, Mari JJ, Andrade LH, Almeida-Filho N, Blay SL, Viana MC, Mello AF.La epidemiologia de las enfermedades mentales en Brasil. In: Rodríguez JJ, Kohn R, Aguilar-Gaxiola S, organizadores. Epidemiología de los trastornos mentales en América Latina. Washington DC: Organização Panamericana de Saúde; 2009.

22. Schmidt MI, Duncan BB, Azevedo e Silva G, Menezes AM, Monteiro CA, Barreto SM, Chor D, Menezes PR. Doenças Crônicas não Transmissíveis no Brasil: Carga e Desafios Atuais. Saúde no Brasil 4; 2011; (5): 61-74.

23. United Nations Office on Drugs and Crime (UNODC). World Drug Report 2010. Viena: United Nations Office on Drugs and Crime (UNODC); 2010.

24. Panamerican Health Organization (PAHO). Drug Use Epidemiology in Latin America and the Caribbean: A Public Health Approach. Washington DC: Panamerican Health Organization; 2009.

25. Centro Brasileiro de Informações sobre Drogas. CEBRID-I. Levantamento domiciliar sobre o uso de drogas psicotrópicas no Brasil: estudo envolvendo as 107 maiores cidades do país. São Paulo: Centro Brasileiro de Informações sobre Drogas (CEBRID); 2001.
26. Centro Brasileiro de Informações sobre Drogas. CEBRID-II. Levantamento domiciliar sobre o uso de drogas psicotrópicas no Brasil: estudo envolvendo as 107 maiores cidades do país. São Paulo: CEBRID; 2005.

27. Instituto Brasileiro de Geografia e Estatística (IBGE). Pesquisa da Assistência Médico-Sanitária - 2009. Rio de Janeiro: Instituto Brasileiro de Geografia e Estatística (IBGE); 2011.

28. Brasil. Ministério da Saúde (MS). Portaria n. ${ }^{\circ} 1101 /$ GM de 12 de junho de 2002. Estabelece os parâmetros assistenciais do SUS. Diário Oficial da União 2002; 13 jun.

29. Brasil. Ministério da Saúde (MS). Portaria 336/GM de 19 de Fevereiro de 2002. Estabelece CAPS I, CAPS II, CAPS III, CAPS i II e CAPS ad II. Diário Oficial da União 2002; 22 fev.

30. Brasil. Ministério da Saúde (MS). Portaria 154/GM de 24 de janeiro de 2008. Cria os Núcleos de Apoio à Saúde da Família - NASF. Diário Oficial da União 2008; 25 jan.

31. Instituto Brasileiro de Geografia e Estatística (IBGE). Pesquisa Nacional por Amostra de Domicílios - PNAD 2008. Suplemento Saúde. Rio de Janeiro: Instituto Brasileiro de Geografia e Estatística (IBGE); 2008.

32. Instituto Brasileiro de Geografia e Estatística (IBGE). Pesquisa da Assistência Médico-Sanitária - 2002. Rio de Janeiro: Instituto Brasileiro de Geografia e Estatística (IBGE); 2005.

33. LaForgia GM, Couttolenc BF. Desempenho Hospitalar no Brasil: Em Busca da Excelência. São Paulo: The World Bank, IBEDESS, Editora Singular; 2009.

34. Brasil. Ministério da Saúde (MS). Relatório Final: IV Conferência Nacional de Saúde Mental Intersetorial. Brasília: Conselho Nacional de Saúde, Ministério da Saúde (MS); 2010.

35. Alves DS. Integralidade nas Políticas de Saúde Mental. In: Pinheiro R, Mattos R. Os Sentidos da Integralidade. Rio de Janeiro: Editora UERJ; 2001.
Apresentado em 08/09/2011

Aprovado em 30/09/2011

Versão final apresentada em 05/10/2011 